\title{
Generation of parallel electric fields in the Jupiter-Io torus wake region
}

\author{
R. E. Ergun, ${ }^{1,2}$ L. Ray, ${ }^{1,2}$ P. A. Delamere, ${ }^{1}$ F. Bagenal, ${ }^{1,2}$ V. Dols, ${ }^{1}$ and Y.-J. $\mathrm{Su}^{3}$
}

Received 8 December 2008; revised 5 February 2009; accepted 10 February 2009; published 2 May 2009.

[1] Infrared and ultraviolet images have established that auroral emissions at Jupiter caused by the electromagnetic interaction with Io not only produce a bright spot, but an emission trail that extends in longitude from Io's magnetic footprint. Electron acceleration that produces the bright spot is believed to be dominated by Alfvén waves whereas we argue that the trail or wake aurora results from quasi-static parallel electric fields associated with large-scale, field-aligned currents between the Io torus and Jupiter's ionosphere. These currents ultimately transfer angular momentum from Jupiter to the Io torus. We examine the generation and the impact of the quasi-static parallel electric fields in the Io trail aurora. A critical component to our analysis is a current-voltage relation that accounts for the low-density plasma along the magnetic flux tubes that connect the Io torus and Jupiter. This low-density region, $\sim 2 R_{J}$ from Jupiter's center, can significantly limit the field-aligned current, essentially acting as a "high-latitude current choke." Once parallel electric fields are introduced, the governing equations that couple Jupiter's ionosphere to the Io torus become nonlinear and, while the large-scale behavior is similar to that expected with no parallel electric field, there are substantial deviations on smaller scales. The solutions, bound by properties of the Io torus and Jupiter's ionosphere, indicate that the parallel potentials are on the order of $1 \mathrm{kV}$ when constrained by peak energy fluxes of a few milliwatts per square meter. The parallel potentials that we predict are significantly lower than earlier reports.

Citation: Ergun, R. E., L. Ray, P. A. Delamere, F. Bagenal, V. Dols, and Y.-J. Su (2009), Generation of parallel electric fields in the Jupiter-Io torus wake region, J. Geophys. Res., 114, A05201, doi:10.1029/2008JA013968.

\section{Introduction}

[2] Jupiter displays several types of auroral processes [Connerney et al., 1993; Clarke et al., 1996, 1998, 2002; Grodent et al., 2003] that include, from lowest to highest latitudes, satellite-driven aurora (spots), a steady, corotationdriven aurora (the main oval), and highly variable auroral activity in the polar regions connected to the outer magnetosphere and possibly influenced by the solar wind. The most conspicuous of the satellite-driven aurora is from Io (Figure 1) which displays a bright spot with a trail of lowerlevel emissions extending eastward (here also called the wake aurora). We focus on the plasma processes that may produce the wake aurora, expanding the model put forth by Delamere et al. [2003] and Su et al. [2003]. Their basic idea is that the interaction between the Io torus and Jupiter's ionosphere on the Io magnetic flux tube is dominated by Alfvén waves [Crary, 1997; Chust et al., 2005] whereas the

\footnotetext{
${ }^{1}$ Laboratory for Atmospheric and Space Physics, University of Colorado, Boulder, Colorado, USA.

${ }^{2}$ Also at Department of Astrophysical and Planetary Sciences, University of Colorado, Boulder, Colorado, USA.

${ }^{3}$ Department of Physics, University of Texas at Arlington, Arlington, Texas, USA.

Copyright 2009 by the American Geophysical Union. 0148-0227/09/2008JA013968
}

interaction that causes the extended trail of emissions in the wake is governed by a large-scale current system driven by subcorotating plasma in the Io torus [Hill and Vasyliunas, 2002].

[3] Figure 1 (top) is a Jovian auroral image taken by the NASA Hubble Space Telescope [after Clarke et al., 2002, Figure 1b]. The brightest emissions of the Io-induced aurora are at the left and believed to be at the base of the Io magnetic flux tube. A faint emission trail extends eastward. Spectral observations from the Space Telescope Imaging Spectrograph combined with a Jovian atmosphere model suggest that the mean electron energies drop from $\sim 70 \mathrm{keV}$ at the footprint to $\sim 40 \mathrm{keV}$ at $20^{\circ}$ downstream of the Io footprint [Gérard et al., 2002]. More recent analysis suggests that the downstream mean energies may be considerably lower (J.-C. Gérard, private communication, 2007). These observations along with the analysis of $\mathrm{Su}$ et al. [2003] suggest the formation of parallel electric fields associated with a large-scale current system in Io's wake.

[4] We focus on a region in the wake (Figure 1, bottom) far enough from Io that the initial Alfvénic perturbation has relaxed. The coordinate system used throughout this article is displayed in Figure 1. We begin with a simple, baseline model of Io's wake which contains a region of subcorotating plasma from Io's interaction with the Io torus. This simple model does not include parallel electric fields; the 

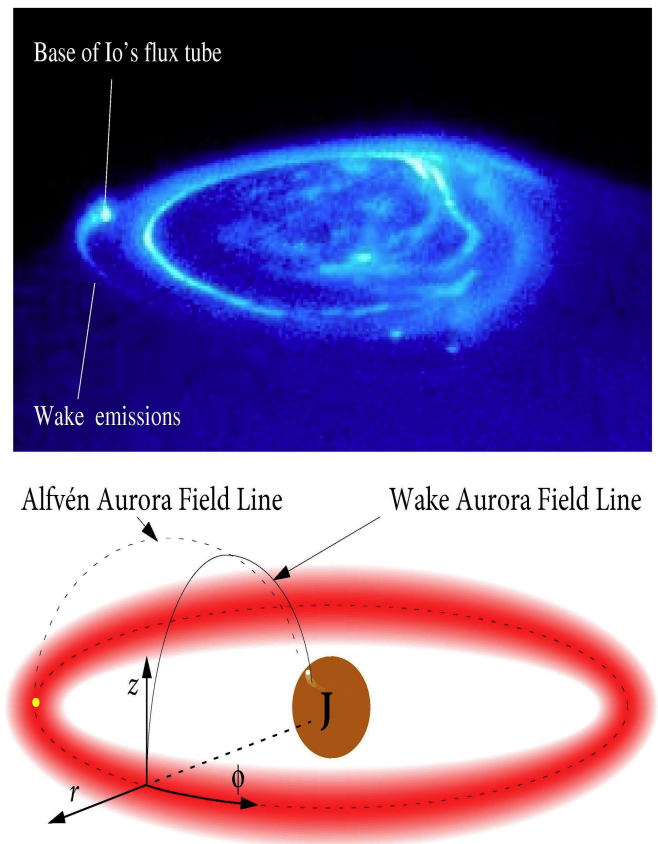

Figure 1. (top) A Jovian auroral image taken by HST [after Clarke et al., 2002, Figure 1b], where the Io-induced aurora is seen at the left with the brightest emissions at the base of the Io flux tube. An emission trail extends eastward. (bottom) The coordinate system used in this article.

momentum exchange between Jupiter and the subcorotating plasma is regulated by the ionospheric conductivity [Hill, 1979; Pontius and Hill, 1982; Hill and Vasyliunas, 2002]. We adopt a symmetric dipole magnetic field to simplify the mathematics. The results of our model are in agreement with that of Hill and Vasyliunas [2002] which predicts that the subcorotating plasma will exponentially relax back to corotation on a timescale of roughly an hour, consistent with the observation of the extended trail in longitude.

[5] Next, we introduce parallel electric fields into the model which requires steady state application of Faraday's law and a current-voltage relation between the Io torus and Jupiter's ionosphere. A current limit is seen on field lines where the current flows from Jupiter's ionosphere to the Io torus [Su et al., 2003; Ray et al., 2009]. Electron flow, which carries the field-aligned currents, is restricted by a combination of low plasma density and magnetic mirror force at $\sim 2 R_{J}$ from Jupiter's center. This restriction in parallel current flow gives rise to a parallel potential on the order of $\sim 1 \mathrm{kV}$ and energy flux into Jupiter's ionosphere on the order of $1 \mathrm{~mW} \mathrm{~m} \mathrm{~m}^{-2}$. The governing equations exhibit some nonlinear behavior which changes the detailed shape of the subcorotating plasma. The timescale on which the plasma is brought back to corotation, however, remains nearly the same as that with no parallel electric field.

[6] The last part of the study examines the behavior of a more realistic profile of subcorotating plasma derived from Galileo observations [Bagenal, 1997] and from simulations [Delamere et al., 2003]. The more realistic profile results in a slightly more intense aurora with a higher potential drop and higher energy flux, both by less than a factor of two.
Thus, within the uncertainties of the input, the model is able to describe the basic physics of the Io wake aurora.

\section{A Simple Model of Io's Wake}

[7] The plasma interaction near Io has been discussed by Saur [2004, and references therein] who point out that an Alfvén wave at Io can be energized by the ion pickup [Goertz, 1980] and charge exchange, as well as by the currents generated by Io's motion [Goldreich and LyndenBell, 1969]. Delamere et al. [2003] proceed to divide the IoJupiter interaction into three phases: an initial mass loading interaction, acceleration of plasma in the wake of Io, and steady state decoupling. The first two phases induce an Alfvénic disturbance which may be responsible for the emissions at Io's magnetic footprint [Crary, 1997; Chust et al., 2005; Ergun et al., 2006; Su et al., 2006]. Roughly 10-20 $R_{I o}$ eastward of Io (Figure 1, wake aurora), the momentum flux from Io's interaction $\left(\dot{P} \sim 1.1 \times 10^{7} \mathrm{~kg} \mathrm{~m}\right.$ $\mathrm{s}^{-2}$, part from mass loading, part from charge exchange, and part from the currents at Io) has rapidly transferred to the torus plasma along the magnetic field $(\boldsymbol{B})$ by Alfvén waves [Delamere et al., 2003]. Far less momentum is transferred transverse to $\boldsymbol{B}$. This momentum transfer results in a region of subcorotating plasma that extends a little over 2 Io radii $\left(R_{I o}\right)$ radially but reaches the edge of the Io torus vertically ( $z$ direction, Figure 2).

[8] The velocity of the subcorotating plasma can be estimated from momentum conservation between the momentum flux from Io $(\dot{P})$ and the momentum flux of the Io torus flux tube:

$$
\dot{P}_{T} \cong m_{i} u_{I o}^{2} \int_{-R_{I o}}^{R_{I o}} \int_{-R_{T}}^{R_{T}} n_{T}(r, z) d z d r \approx 1.2 \times 10^{8} \mathrm{~kg} \mathrm{~ms}^{-2}
$$

where $m_{i}$ is the average ion mass ( $~ 20$ AMU), $u_{I o}$ is the (positive) relative velocity between the corotation and Io ( $57 \mathrm{~km} \mathrm{~s}^{-1}$ in $\varphi$ direction), $n_{T}$ is the plasma density, and $R_{T}$ is the vertical $(z)$ extent of the Io torus. Here, curvature of the magnetic field is ignored. The velocity of the subcorotating plasma in a frame that corotates with Jupiter is:

$$
u=\frac{-\dot{P}}{\dot{P}_{T}+\dot{P}} u_{I o}
$$

Using a representative density model of the Io torus [Bagenal, 1994], the subcorotation velocity predicted by equation (2) is roughly $5 \mathrm{~km} / \mathrm{s}$, in agreement with hybrid simulation results [Delamere et al., 2003]. The potential generated by the subcorotating plasma, $\sim 30 \mathrm{kV}$ over $2 R_{I o}$ in $r$, maps to the ionosphere and drives ionospheric currents. The ionospheric currents, in turn, set up field-aligned currents between Jupiter and the Io torus in Io's wake. These currents ultimately are responsible for transferring momentum that brings the Io torus nearly back to corotation [Hill and Vasyliunas, 2002].

[9] We model the momentum transfer by examining the interaction in the $r-z$ plane at a fixed value of $\varphi$ and time. The model is similar to that of Hill and Vasyliunas [2002], with the addition of the radial profile. We treat the ionosphere as a thin resistive layer magnetically connected to the Io torus. We also assume that the subcorotating plasma is slowly brought back to corotation, so variations in time and 

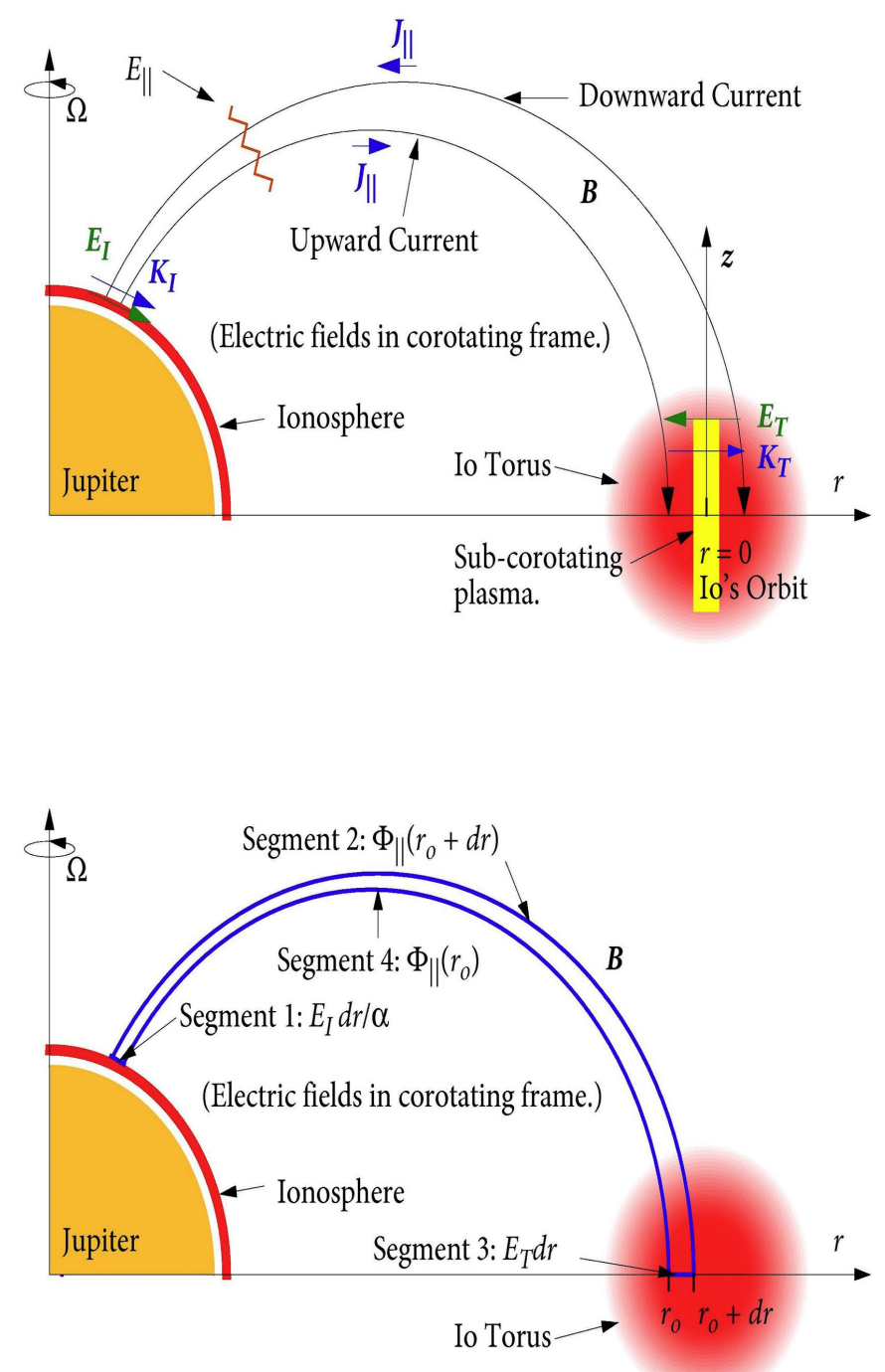

Figure 2. (top) A diagram of the current system between Jupiter's ionosphere and the Io torus. (bottom) The potentials on two closely spaced (in radius) field lines. Faraday's law implies that the ionospheric electric field must differ from the torus electric field to build a quasi-static parallel potential.

in the $\varphi$ direction are small. This latter assumption is in consort with the large longitudinal extent of the wake aurora [Clarke et al., 1996] and will be justified mathematically later. The quantities in the Io torus are height-integrated.

[10] The electric field in the Io torus in a frame corotating with Jupiter can be described by:

$$
E_{T}=u \times B_{T}
$$

where $u$ is the subcorotation velocity in the $\varphi$ direction and $B_{T}$ is the magnetic field in the torus, assumed to be in the $-z$ direction. $E_{T}$ maps to the ionosphere as:

$$
E_{I}=\alpha E_{T}
$$

where the parameter $\alpha$ depends on the magnetic field geometry. Here, we assume a dipole magnetic field. The height-integrated current in the ionosphere is expressed as:

$$
K_{I}=\Sigma_{P} E_{I}
$$

with $\Sigma_{P}$ representing the height-integrated Petersen conductivity. The ionospheric current closes in the Io torus resulting in a z-integrated current:

$$
K_{T}=-2 \beta K_{I}
$$

The factor of two assumes identical response from both hemispheres and $\beta$, as does $\alpha$, represents a mapping factor that depends on the magnetic field mapping.

[11] Equations (3)-(6) form a closed set describing the quasi-static interaction between the Io torus and Jupiter. To solve these equations, an initial velocity profile in the Io torus must be defined. We start with a smoothed rectangular function (boxcar function) of subcorotating plasma with a subcorotation velocity of $5 \mathrm{~km} / \mathrm{s}$ and a width of $2 R_{I o}$. The smoothing is needed so that parallel currents are finite.

[12] Figure 3 shows the electric fields (Figure 3a), the parallel currents as would be measured in the ionosphere $\left(J_{\|}^{I}\right.$, Figure $\left.3 b\right)$, and the height-integrated, radial current in the Io torus (Figure 3c) as a function of radial distance from Io's orbit. This slice in the radial direction (horizontal axis) is at a value $\varphi$ far enough from Io (say, $\sim 10 R_{I o}$, Figure 1) 


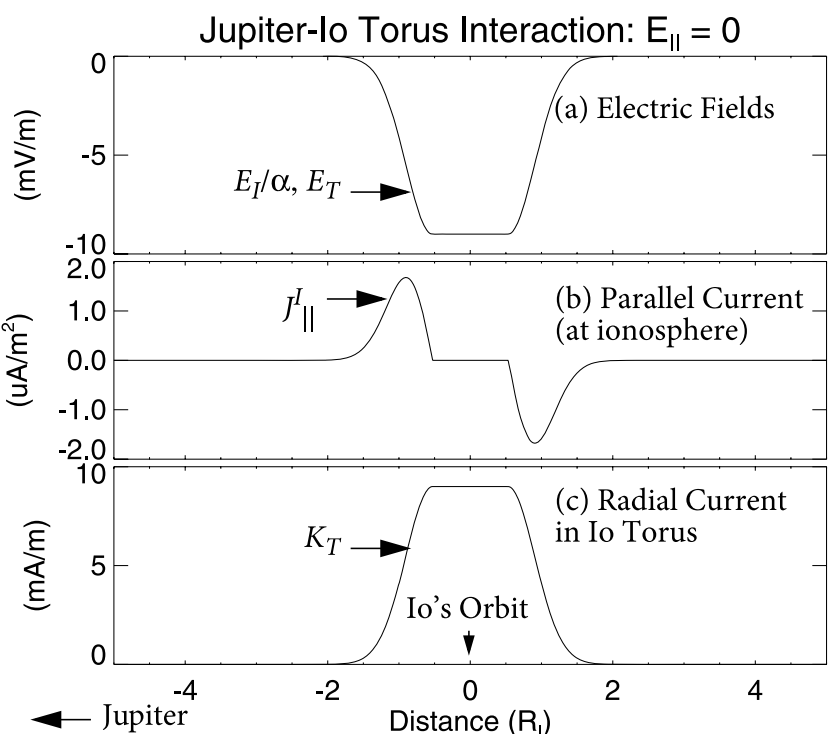

Figure 3. The results of a simple model with no parallel electric fields. (a) Ionospheric and magnetospheric electric fields, (b) field-aligned currents, and (c) the heightintegrated radial current in the Io torus as a function of radial position from Io's orbit.

so that the steady state assumption is valid. The ionospheric electric field and the magnetospheric electric field map ideally (equation (4)) resulting with an upward current (current from Jupiter to the Io torus) on the Jovian side of the Io torus and a downward current on the outside of the Io torus. The shape of the radial current in the Io torus (Figure 3c) is identical to that of the electric field (Figure 3a).

[13] Combining equations (3)-(6), and introducing a slow time variation, the force exerted on the subcorotating plasma can be approximated as [see Hill and Vasyliunas, 2002, equation (5)]:

$$
\begin{aligned}
& \rho_{T} \frac{\partial u_{s}}{\partial t}=K_{T} \times B_{T}=-2 \alpha \beta \Sigma_{P} B_{T}^{2} u_{s}, \\
& \rho_{T} \cong m_{i} \int_{-R_{T}}^{R_{T}} n_{T}(r, z) d z
\end{aligned}
$$

[14] Here, $\rho_{T}$ is the height-integrated mass density of the Io torus. The above equation has the simple solution $u_{S}=$ $u_{o} e^{-t / \tau}$ where $\tau=\rho_{T} /\left(2 \alpha \beta \Sigma_{P} B_{T}^{2}\right)$ indicating that the initially subcorotating plasma is brought back to corotation by exponential relaxation [Hill and Vasyliunas, 2002]. Using canonical values of the torus, $\rho_{T} \sim 10^{-8} \mathrm{~kg} \mathrm{~m}^{-2}$ [Bagenal, 1994], $B_{T} \sim 1800 \mathrm{nT}$ [Kivelson et al., 1996], $\Sigma_{P} \sim 0.5 \Omega^{-1}$ [Hill, 1979; Millward et al., 2002], and $\alpha \beta \sim 1.3$ (dipole), the relaxation time is $\sim 1 \mathrm{~h}$, consistent with the visible extension of the wake aurora [Clarke et al., 2002; Hill and Vasyliunas, 2002].

[15] Solutions with several values of $\Sigma_{P}$ are plotted in Figure 4 . The above results are analytically nearly identical to those of Hill and Vasyliunas [2002]. Differences in relaxation times are from use of different torus density profiles and ionospheric conductivities, both of which, arguably, have significant uncertainties. In any case, the relaxation time is many times the Alfvén propagation time from the Io torus to Jupiter so our assumption that time variations and $\varphi$ variations are small is justified. Furthermore, since the governing equations ((3)-(6)) are linear, the relaxation is self-similar; the shape (normalized profile in $r$ ) of the subcorotating plasma does not change as the amplitude declines.

\section{Modeling Parallel Electric Fields}

[16] In this section, we examine a parallel electric field that develops in the upward current region (current flowing from Jupiter). We assume that no parallel electric field develops in the downward current region. At Earth, the parallel potential in the upward current region is typically about five to tens times of that in the downward current region. The upward current regions have the mirror force acting against the primary current carriers, electrons [Knight, 1973; Paschmann et al., 2003], whereas the downward current regions do not. A study of the parallel potential of the downward current region would be an interesting extension of this work.

[17] The inclusion of parallel electric fields alters the detailed solution from that developed above. The set of governing equations are nonlinear and the resulting solutions do not create a self-similar relaxation. Rather, the upward current at the inner edge of the subcorotating plasma is spatially spread over a larger region. The model requires the derivation of a current-voltage relation for the field lines that connect the Io torus to Jupiter, a modification of equation (4) to properly map electric potentials, and a current continuity equation to close the set of equations.

\subsection{Current-Voltage Relation}

[18] The electromagnetic interaction between the Io torus and Jupiter transpires through nearly vacant magnetic flux tubes connecting the plasma in Jupiter's ionosphere to the plasma bound by centrifugal force to the Io torus. This region was examined in detail with a static Vlasov code by Su et al. [2003], who concluded that the current-voltage relation at Jupiter was dominated by the low-density plasma $\sim 2 R_{J}$ (Jovian Radii) from Jupiter's center. More specifically, a current-voltage relation based on equatorial plasma conditions [Knight, 1973; Paschmann et al., 2003] was

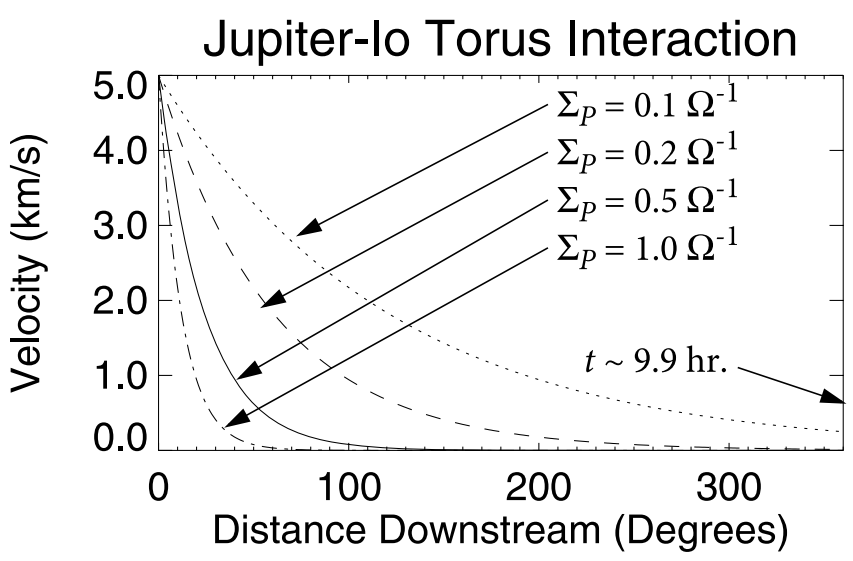

Figure 4. The corotation velocity at $r=0$ (Io's orbit) as a function of azimuth (or time). The line styles indicate solutions under various values of $\Sigma_{P}$. 
found to be not applicable, primarily because of the low densities in the high-latitude magnetosphere. Here, we adopt a modified analytical model that is in close agreement with an analytical study [Boström, 2003] and to the results of the static kinetic Vlasov model [Ergun et al., 2000; Su et al., 2003; Ray et al., 2009] that was used to model the Jupiter-Io torus upward current region. The current-voltage relation is expressed as a mirror resistance [Boström, 2003; Ray et al., 2009]:

$$
J_{\|}^{I}=J_{\|} R_{T}=j_{x}+j_{x}\left(R_{x}-1\right)\left(1-e^{-\left(\frac{e \Phi_{\|}}{T_{x}\left(R_{x}-1\right)}\right)}\right)
$$

where $J_{\|}^{I}$ is the current density at Jupiter's ionosphere, $J_{\|}$is the current density at the Io torus, $R_{T}$ is the magnetic mirror ratio between the torus and Jupiter's ionosphere, $j_{x}=e n_{x}\left(T_{x} /\right.$ $\left.2 \pi m_{e}\right)^{1 / 2}$ is the electron thermal current, $R_{x}$ is the magnetic mirror ratio, $T_{x}$ is the electron temperature (expressed in units of energy), $n_{x}$ is the electron density, $m_{e}$ is the electron mass, and $e$ is the fundamental charge. This expression is very near that derived by Boström [2003] but accounts for a hot electron population in the Io torus. It also is similar to Earth models [Knight, 1973; Paschmann et al., 2003], but is based on plasma conditions at a point that has the lowest thermal current along the magnetic flux tube. The subscript $(x)$ thus indicates that the quantities are evaluated at the location along the flux tube between the ionosphere and magnetosphere where $j_{x}$ is a minimum. Equation (8) is valid only for $J_{\|}^{I} \geq 0$, otherwise we assume $\Phi_{\|}=0$. On auroral flux tubes at Earth, $j_{x}$ is at its minimum in the plasma sheet. At Jupiter, the minimum is at $\sim 2 R_{J}$ from Jupiter's center [Su et al., 2003; Ray et al., 2009]. The plasma parameters at $\sim 2 R_{J}$ dramatically differ from those in the Io torus, so this modified current-voltage relation is of critical importance. Without this modified current-voltage relation, no significant parallel electric fields are expected to form; essentially, the field-aligned conductance using a uniform density and mirror force [Knight, 1973; Paschmann et al., 2003] is nearly two orders of magnitude higher than the modified version. We call this modified current-voltage relation the "high-latitude current choke."

\subsection{Development of Parallel Potential}

[19] If one considers the loop outlined in Figure 2 (bottom), Faraday's law demands that:

$$
\oint E \cdot d l=\int_{S} \frac{\partial B}{\partial t} \cdot d A
$$

The relaxation timescale of the subcorotating plasma, and therefore that of the field-aligned currents, roughly several hours, is such that the term $\partial B / \partial t$ is negligible (certainly, $\partial B / \partial t$ is not negligible close to Io where Alfvén waves dominate). Therefore, the sum of the segments in Figure 2 (bottom) must be zero. Defining $E_{T}$ to be positive in the $r$ direction, $E_{I}$ positive if toward the pole, and $\Phi_{\|}$, the potential between the ionosphere and Io torus, to be positive if the Io torus is at higher potential:

$$
\frac{E_{I} d r}{\alpha}=E_{T} d r-\Phi_{\|}\left(r_{o}+d r\right)+\Phi_{\|}\left(r_{o}\right)
$$

which implies:

$$
E_{I}=\alpha\left(E_{T}-d \Phi_{\|} / d r\right)
$$

A gradient in, not the value of, a parallel potential requires that the ionospheric electric field and Io torus electric field not map to each other [Lyons et al., 1979; Lyons, 1980, 1981].

\subsection{Governing Equations}

[20] To complete the modified set of equations, we express the relation between $J_{\|}$and $K_{T}$ as (current continuity):

$$
J_{\|}(r)=\frac{1}{r+a_{I o}} \frac{d}{d r}\left(r K_{T}\right)
$$

where $a_{I o}$ is the orbital distance of Io from Jupiter's center. Equations (3), (5), (8), (11), and (12) represent a closed set which include parallel potentials generated by field-aligned currents. The symbols are summarized in Table 1 .

[21] For the purpose of this article, the ionospheric conductivity, $\Sigma_{P}$, is estimated to be about $\sim 0.5 \Omega^{-1}$ at high latitude. This value is not well established, so we will treat $\Sigma_{P}$ as a parameter. It, however, can change with precipitating electron flux. In particular, $\Sigma_{P}$ will depend on the energy flux and the characteristic energy of electrons incident on Jupiter's ionosphere. We use a simple model similar to that used by Millward et al. [2002] and Nichols and Cowley [2003, 2004]:

$$
\Sigma_{P}=\Sigma_{P o}\left(1+\left(C_{o} J_{\|} \Phi_{\|}\right)^{\gamma}\right)
$$

where $\Sigma_{P o}$ is the height-integrated Pedersen conductivity in nonauroral regions and $C_{o}$ and $\gamma$ are estimated parameters. For the initial solutions, we set $\Sigma_{P o}=0.5 \Omega^{-1}, C_{o}=0.1$ $\mathrm{W}^{-1} \mathrm{~m}^{2}$, and $\gamma=1 / 2$ [Millward et al., 2002]. In this work, we find that the conductivity change is small and does not dramatically affect the solutions.

\section{Numerical Solutions}

[22] As with most models, there are several of parameters that are but loosely constrained, in particular $n_{x}, T_{x}$ and the initial profile of the subcorotating plasma, and there is observational evidence that can test the model and, subsequently, somewhat constrain the input. The most salient observational evidence is the energy flux of $\sim 1 \mathrm{~mW} \mathrm{~m}^{-2}$ [Clarke et al., 2002] derived from ultraviolet images and the extent in latitude $(\sim 1000 \mathrm{~km})$ [Clarke et al., 1996]. The mean energy of the precipitating electrons was reported to be roughly $40 \mathrm{kV}$ [Gérard et al., 2002]. However, a more recent analysis of the UV emission from Io's trail aurora indicates that the mean energy of the precipitating electrons may be significantly lower than the earlier results, so we do not constrain the parallel potential in our model.

\subsection{Baseline Solution}

[23] The high-latitude electron density and temperature are estimated from the numerical results of $\mathrm{Su}$ et al. [2003] and Ray et al. [2009]. We choose $n_{x} \sim 0.5 \times 10^{6} \mathrm{~m}^{-3}$ and $T_{x} \sim 100 \mathrm{eV}$ as baseline values. The effective mirror ratio is 
Table 1. Symbols

\begin{tabular}{|c|c|c|c|}
\hline Symbol & Description & Type & Units \\
\hline$\alpha(r)$ & Magnetosphere-ionosphere radial magnetic field mapping. & Prescribed & \\
\hline$\beta$ & Magnetosphere-ionosphere tangential magnetic field mapping. & Prescribed & \\
\hline$B_{T}(r)$ & Magnetic field in Io torus. & Prescribed & $\mathrm{T}$ \\
\hline$E_{I}(r)$ & Ionospheric electric field (North). In corotating frame. & Variable & $\mathrm{V} \mathrm{m} \mathrm{m}^{-1}$ \\
\hline$E_{T}(r)$ & Io torus electric field (radial). In corotating frame. & Prescribed & $\mathrm{V} \mathrm{m} \mathrm{m}^{-1}$ \\
\hline$\Phi_{\|}(r)$ & Parallel potential between ionosphere and magnetosphere. & Variable & $\mathrm{V}$ \\
\hline$J_{\|}^{\|}(r)$ & Field-aligned current density in the magnetosphere. & Variable & $\mathrm{A} \mathrm{m}^{-2}$ \\
\hline$K_{I}(s)$ & Height-integrated current (ionosphere). & Variable & $\mathrm{A} \mathrm{m} \mathrm{m}^{-1}$ \\
\hline$K_{T}(r)$ & Height-integrated current (Io torus). & Variable & $\mathrm{A} \mathrm{m} \mathrm{m}^{-1}$ \\
\hline$\dot{P}$ & Io's momentum exchange with the Io torus. & Prescribed & $\mathrm{kg} \mathrm{m} \mathrm{s}^{-2}$ \\
\hline$r$ & Radial position in Io torus. & Ordinant & $\mathrm{m}$ \\
\hline$s$ & Corresponding position in Jupiter's ionosphere. & Ordinant & $\mathrm{m}$ \\
\hline$R_{T}(r)$ & Magnetic mirror ratio at the Io torus $=\mathrm{a} / \mathrm{b}$. & Prescribed & \\
\hline$\rho_{T}$ & Height-integrated mass density in the Io torus. & Prescribed & $\mathrm{kg} \mathrm{m}^{-2}$ \\
\hline$\Sigma_{\mathrm{P}}$ & Height-integrated Petersen conductivity. & Variable & $\Omega^{-}$ \\
\hline
\end{tabular}

$R_{x} \sim 8$ assuming the lowest value of $j_{x}$ is at $\sim 2 R_{J}$ from Jupiter's center. As earlier, the initial runs use a smoothed rectangular function (boxcar function) of subcorotating plasma with a subcorotation velocity of $5 \mathrm{~km} / \mathrm{s}$ and a width of $2 R_{I o}$.

[24] The model starts with an imposed velocity profile $\left(u_{T}\right)$ which also imposes $E_{T}$. The solution to equations (3), (5), (8), (11), and (12) is obtained though use of a combination of two numerical methods. Newton's method (advancing in $r$ from $r=-10 R_{I o}$ with differentiation) is used when $J_{\|}^{I} \ll J_{x}$. Once $J_{\|}^{I}$ becomes a significant fraction of $J_{x}$, Newton's method becomes numerically unstable so direct integration is used (which is not possible if $J_{\|}^{I} \sim 0$ ). Because the equation set is nonlinear, the solutions require both boundaries $\left(r= \pm 10 R_{I o}\right)$ to be fixed. As the code changes from Newton's method to direct integration, a small offset $(\sim 1 \mathrm{mV})$ in $\Phi_{\|}$is introduced. The offset is adjusted through iteration until the boundary condition at $r=10 R_{I o}$ is met. Identical solutions were found by negative advancement from the outer boundary and through guessing the peak potential and advancing in both directions from the point of maximum potential.

[25] Figure 5 presents a radial view of the solution. The horizontal axis is radial distance from Io's orbit; positive is away from Jupiter. Figure 5a displays the imposed electric field at the Io torus (dashed line, also represents the subcorotating plasma velocity) and the resulting electric field at Jupiter's ionosphere (solid line) mapped to the Io torus. The integrated difference between the two traces $\left(E_{I} / \alpha\right.$ and $E_{T}$ ), represented by the shaded areas ' $\mathrm{A}$ ' and ' $\mathrm{B}$ ', is the field-aligned potential (equation (11)) plotted in Figure $5 \mathrm{~b}$. The areas ' $\mathrm{A}$ ' and ' $\mathrm{B}$ ' must be identical to obtain a valid solution. The peak in the potential is on the Jovian side of the subcorotating plasma where the upward current is driven. Our model predicts parallel potentials of $\sim 1 \mathrm{kV}$ which are far lower than the potentials derived from analysis of UV emissions combined with atmospheric modeling [Gérard et al., 2002].

[26] Figure 5c plots $J_{\|}^{I}$ (as mapped to the ionosphere) as a solid line. For comparison, the dashed line represents $J_{\|}^{I}$ if no parallel electric fields were in the model (same as in Figure 3). Figure 5d plots the energy flux into the ionosphere which is in agreement with UV observations [Clarke et al., 2002], Figure 5e plots the radial current in the Io torus, and Figure $5 \mathrm{f}$ plots the ionospheric conductivity.
The change in ionospheric conductivity is small (it cannot be seen in the plot) and does not significantly alter the solutions.

[27] The nonideal mapping of $E_{I}$ and $E_{T}$ is of great importance in modeling parallel electric fields as they force a nonlinear, and sometimes a somewhat nonintuitive, response. Because of limitation on parallel currents, the Jovian ionosphere is forced to subcorotate at $r<-R_{I o}$, even though the Io torus is in near corotation. This behavior can be best discussed by linearizing equations (3), (5), (8), (11), and (12) and assuming that $E_{T}=0$ (or constant). Equation (10) becomes $E_{I}=-\alpha\left(d \Phi_{\|} / d r\right)$. To simplify the mathematics, we approximate the current-voltage relation (equation (8)) as $J_{\|}^{\prime}=\sigma_{\|} \Phi_{\|}$where $\sigma_{\|}=e^{2} n_{x} /\left(2 \pi m_{e} T_{e}\right)^{-1 / 2}$. Last, we approximate equation (11) as $J_{\|}(r)=d K_{T} / d r$.

[28] Combining the three above equations with equations (5) and (6) and treating $\alpha, \beta, \sigma_{\|}$, and $\Sigma_{P}$ as constants, one can show:

$$
\frac{d^{2} \Phi_{\|}}{d r^{2}}=\frac{\sigma_{\|}}{2 \alpha \beta R_{T} \Sigma_{P}} \Phi_{\|}
$$

which has the solution $\left(E_{I}\right.$ as a function of magnetic position in Io torus) $\Phi_{\|}=\Phi_{o} e^{ \pm r / r o}$ with:

$$
r_{o}=\sqrt{2 \alpha \beta R_{T} \Sigma_{P} / \sigma_{\|}}
$$

This previously derived result [Lyons, 1980] establishes a transverse radial scale size of an auroral arc driven by a quasi-static parallel electric potential. The above scale size includes the mirror ratio since it applies to the Io torus. In Jupiter's ionosphere, the scale size is $r_{o}^{I}=\sqrt{2 \sum_{P} / \sigma_{\|}}$ Using the same model parameters as earlier, $\Sigma_{P}=0.1 \Omega^{-1}$ and $\sigma_{\|} \sim 10^{-10} \Omega^{-1} \mathrm{~m}^{-2}$ [Paschmann et al., 2003, equation (3.38)], $r_{o} \sim R_{I o}$. This result is consistent with the scale size of $J_{\|}^{J}$ seen in Figure $5 \mathrm{~d}(-2.5<r<-1.5)$.

[29] Another way of understanding the effect of parallel electric fields is to examine the upward current density $\left(J_{\|}^{I}\right)$. Compared to the case with no parallel electric field (Figure $5 \mathrm{c}$ ), the peak value of $J_{\|}^{I}$ is reduced. However, $J_{\|}^{I}$ is distributed over a larger radial distance so the total current between Jupiter and the Io torus remains nearly unchanged.

[30] In the extreme case of high conductivity, that is, $\sigma_{\|}$is much larger, then $r_{o} \ll R_{I o}$ and no significant parallel 


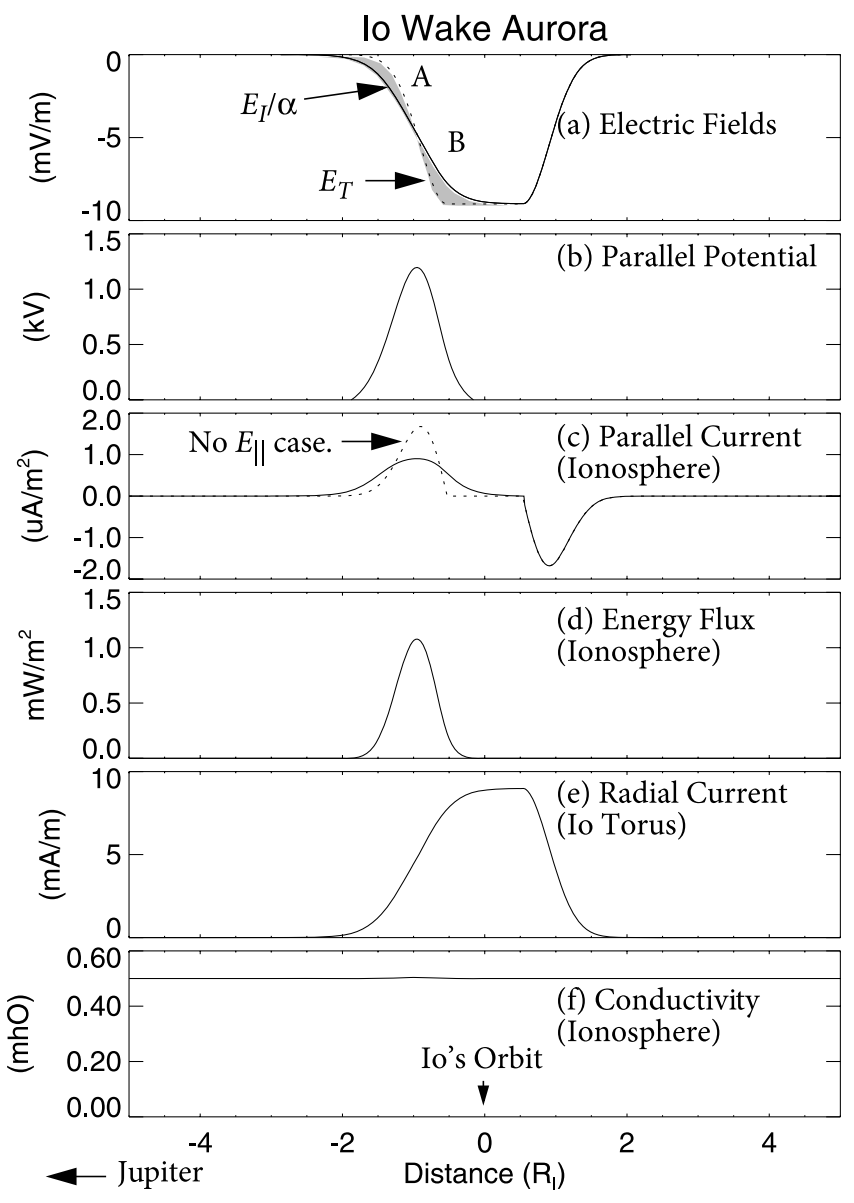

Figure 5. The results of our model with parallel electric fields. Here, $\Sigma_{P}=0.5 \Omega^{-1}, n_{x}=0.5 \times 10^{6} \mathrm{~m}^{-3}$ and $T_{x}=100 \mathrm{eV}$. (a) Ionospheric and magnetospheric electric fields, (b) the parallel potential, (c) field-aligned currents, (d) the electron energy flux into Jupiter's ionosphere, (e) the height-integrated radial current in the Io torus, and (f) the ionospheric conductivity as a function of radial position from Io's orbit.

potentials would form. On the other hand, if $J_{x}$ and $\sigma_{\|}$were smaller, the upward current would be further reduced and even more spread in radial distance; less total upward current would flow. In this latter case, the relaxation time constant, which in the ideal case is dominated by $\Sigma_{P}(\tau=$ $\left.\rho_{T} /\left(2 \alpha \beta \Sigma_{P} B_{T}^{2}\right)\right)$, would be increased. In the extreme case of low conductivity, $\sigma_{\|}$would dominate that time constant rather than $\Sigma_{P}$. In the Io case, the "current choke" at high latitudes is largely responsible for the generation of the wake aurora but does not appear to dominate the relaxation time constant.

\subsection{Long-Term Evolution}

[31] We next examine the long-term evolution of the subcorotating plasma. The solution is iterated by calculating $J \times B$ force in the Io torus from the static solution (Figure 5e) and adjusting the subcorotating velocity profile by $u_{T} \rightarrow u_{T}+\Delta u_{T}$ with (see equation (7)):

$$
\Delta u_{T}=\frac{K_{T} \times B_{T}}{\rho_{T}} \Delta t
$$

where the time step, $\Delta t \sim 500 \mathrm{~s}$, is a chosen to represent $\sim 5^{\circ}$ of Jupiter's rotation. A new quasi-static solution is then found.

[32] Figure 6 displays the evolution of $u_{T}$ as a function of time (and azimuthal angle, $\varphi$ ). In general, the solutions show a near-exponential decay in the energy flux and maximum potential as predicted in the simplest case (no parallel electric fields). However, one can see that the solution with a parallel electric field (Figure 6) does not yield the self-similar decay, rather the outer (anti-Jovian) side of the subcorotating plasma relaxes back to corotation faster. The difference between $E_{I} / \alpha$ and $E_{T}$ (Figure 5a) causes a vortex to form at the inner edge of the subcorotating plasma. After $\sim 60^{\circ}(1.6 \mathrm{~h})$, the vortex (Figure 6 ) is fully formed with a supercorotation velocity of $\sim 0.3 \mathrm{~km} / \mathrm{s}$. It then begins a nearly self-similar decay. After one full Jovian sidereal rotation, a small vortex is still seen, but, when integrated over the radial distance, the net velocity is nearly zero. Less than $1 \%$ of the initial momentum is left in the system.

\section{Parametric Study}

[33] To investigate the effect of poorly constrained input parameters, we rerun the model under a variety of inputs. The most sensitive parameters are the ionospheric conductivity $\left(\Sigma_{P}\right)$, the electron density and temperature at high latitudes $\left(n_{x}\right.$ and $\left.T_{x}\right)$, the subcorotation velocity of the plasma in the Io torus $(u)$, and the radial profile of $u$. In the latter case, we change the smoothing of the initial boxcar to make a wider profile or a more abrupt profile.

[34] Figure 7 displays a run that maximizes the parallel potential. The format of the plot is identical to that of Figure 5. Here, $\Sigma_{P}=1 \Omega^{-1}, n_{x}=0.25 \times 10^{6} \mathrm{~m}^{-3}$ and $T_{x}=50 \mathrm{eV}$. Compared to the baseline solution (Figure $5, \Sigma_{P}=1 \Omega^{-1}, n_{x}$ $=0.5 \times 10^{6} \mathrm{~m}^{-3}$ and $\left.T_{x}=100 \mathrm{eV}\right)$, the limiting effect of the high-latitude current choke dominates over that of $\Sigma_{P .}$ As a result, the ionospheric electric field and magnetospheric electric field are more decoupled (Figure 7a). The parallel potential reaches over $8 \mathrm{kV}$ (Figure $7 \mathrm{~b}$ ) and the energy flux into the ionosphere maximizes at $\sim 3 \mathrm{~mW} \mathrm{~m}^{-2}$. The upward field-aligned current (Figure 7c) is strongly limited causing it to spread over a larger distance.

[35] Table 2 presents the results of a systematic variation in each of the parameters, one at a time, from the baseline case (Figure 5). The peak parallel potential and peak energy flux are sensitive to all of the parameters except the highlatitude electron temperature. An increase in $\Sigma_{P}$ results in a corresponding increase in ionospheric currents and thus the field-aligned currents. As a result, the peak potential increases and, in particular, the peak energy flux increases dramatically. Increasing the high-latitude density creates more current carriers and thus allows for higher current flow with lower parallel potential. An increase in $n_{x}$ results in a significant change in peak parallel potential, but results in a smaller change in energy flux. An increase high-latitude electron temperature creates more current carriers but also increases the mirror resistance (equation (8)). The two nearly offset, so the peak parallel potential and peak energy flux are not highly sensitive to $T_{x}$.

[36] Interestingly, the peak parallel potential and peak energy flux show a very strong dependence on subcorotation velocity, the driver of the system. A factor of two increase in $u$ results in a greater than fivefold increase in 


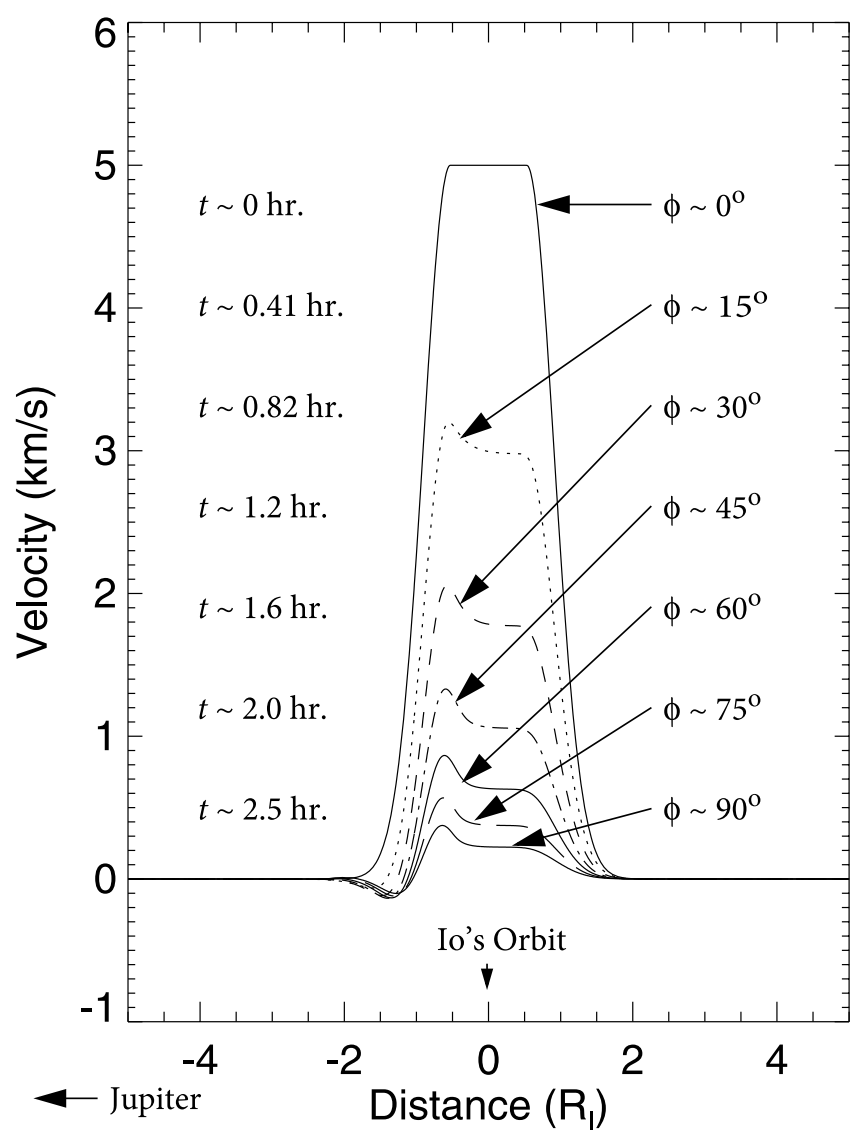

Figure 6. The deviation from corotation velocity at $r=0$ (Io's orbit) as a function of radial distance. The line styles indicate solutions in different locations in $\varphi$ (and time). The shape of the subcorotating plasma changes with $\varphi$ (and time).

peak energy flux. This strong sensitivity results from the nonlinear behavior of the system. The increase in $u$ causes an increase in $E_{T}$ and thus $E_{I}$, forcing larger currents and thus larger parallel potentials. However, it also creates a larger net potential across the subcorotating region in the torus, allowing for an additional increase in parallel potential. The width of the subcorotating region in the torus also can affect the peak parallel potential and peak energy flux. If the subcorotating region spreads over a wide region (keeping the net potential across the subcorotating region constant), the parallel currents are spread over a wider region and thus are not as intense.

\section{Effects of an Incompressible Magnetic Field}

[37] The above analysis highlighted the physics associated with parallel electric fields, but ignores the effects of Jupiter's nearly incompressible magnetic field which brings about finite pressure gradients in the Io torus. The primary effect, the nearly incompressible magnetic field, causes the net radial electric field to be nearly zero in the corotating frame:

$$
\int_{r_{\min }}^{r_{\max }} u_{T} \times B_{T} \approx 0
$$

Thus, the simple profiles we have used as an initial condition are likely to be much more complex; there can be a net mass flow (angular momentum) in the $\varphi$ direction, but there cannot be a net flux of magnetic field. We further our analysis by using a velocity profile in the Io torus derived from Galileo observations [Bagenal, 1997] and from MHD simulation results of the Io-Io torus reaction [Delamere et al., 2003].

[38] Figure 8 plots the solutions to a more realistic velocity profile (smoothed functional fit) that mimics the velocity profile expected $10 R_{I o}$ (in $\varphi$ direction) from Io. All other parameters are identical to those used in the earlier solution. The format of Figure 8 is nearly identical to that of Figure 5. The dashed line in Figure $8 \mathrm{a}$ is the imposed electric field in the torus which is representative of the velocity profile. One can see that the integrated radial electric field is nearly zero whereas the peak flows are nearly identical to the earlier model. The parallel potential (Figure 8b) and the energy flux (Figure 8d) are higher than the baseline case (Figure 5). We emphasize that the values of parallel potential and energy flux are sensitive to the currentvoltage relation, in particular $J_{x}$, the height-integrated mass

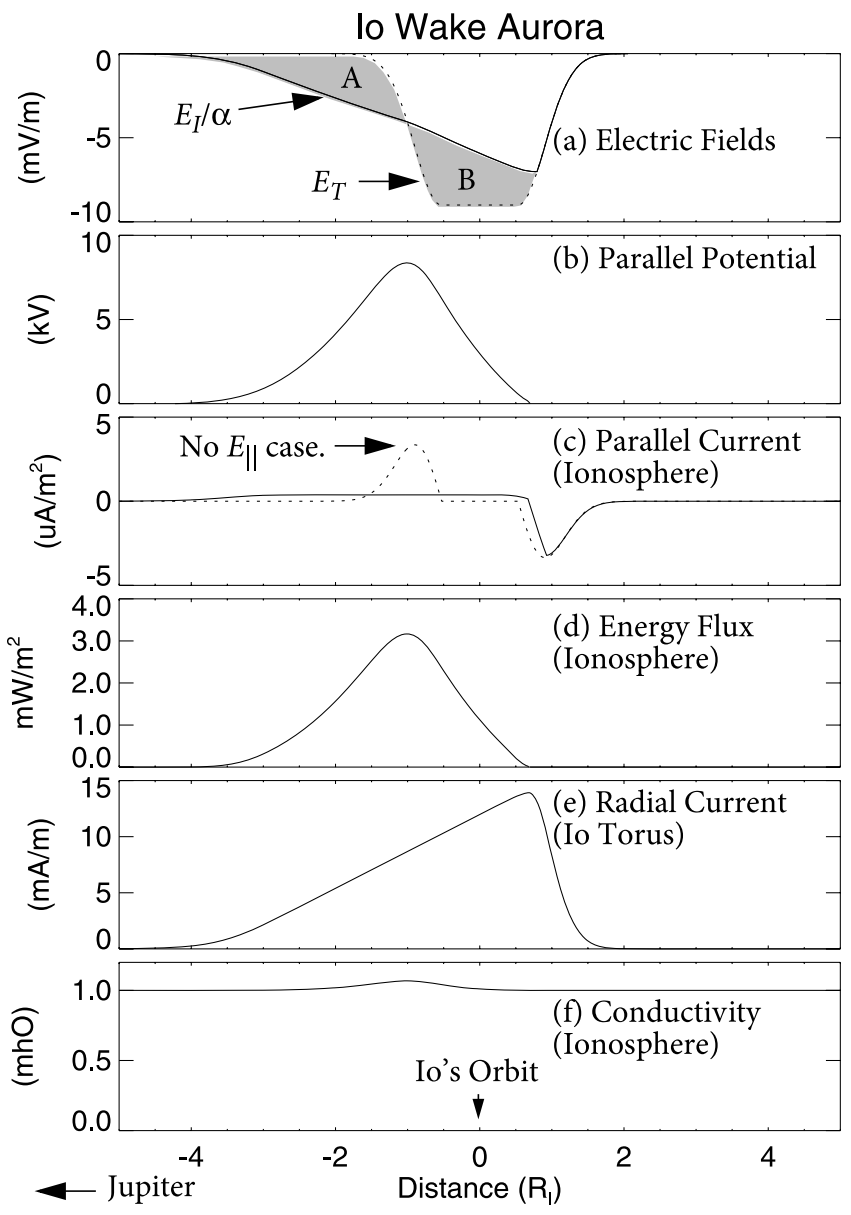

Figure 7. The results of our model with increased ionospheric conductance and decreased field-aligned conductance $\left(\Sigma_{P}=1 \Omega^{-1}, n_{x}=0.25 \times 10^{6} \mathrm{~m}^{-3}\right.$ and $\left.T_{x}=50 \mathrm{eV}\right)$. (a) Ionospheric and magnetospheric electric fields, (b) the parallel potential, (c) field-aligned currents, (d) the electron energy flux into Jupiter's ionosphere, (e) the heightintegrated radial current in the Io torus, and (f) the ionospheric conductivity as a function of radial position from Io's orbit. 
Table 2. Parametric Variations

\begin{tabular}{|c|c|c|c|c|c|}
\hline Parameter & $\Sigma_{P}$ & $n_{\mathrm{x}}$ & $T_{\mathrm{x}}$ & $u_{\max }$ & Width \\
\hline High value & $1.0 \Omega^{-1}$ & $1 \times 10^{6} \mathrm{~m}^{-3}$ & $200 \mathrm{eV}$ & $10 \mathrm{~km} / \mathrm{s}$ & $\sim 4 R_{\mathrm{I}}$ \\
\hline Peak parallel potential $(\mathrm{kV})$ & 2.90 & 0.50 & 1.16 & 5.25 & 0.55 \\
\hline Peak energy flux ( $\left.\mathrm{mW} \mathrm{m}^{-2}\right)$ & 3.06 & 0.60 & 1.08 & 5.62 & 0.36 \\
\hline Baseline & $0.5 \Omega^{-1}$ & $5 \times 10^{5} \mathrm{~m}^{-3}$ & $100 \mathrm{eV}$ & $5 \mathrm{~km} / \mathrm{s}$ & $3 R_{\mathrm{Io}}$ \\
\hline Peak parallel potential $(\mathrm{kV})$ & 1.20 & 1.20 & 1.20 & 1.20 & 1.20 \\
\hline Peak energy flux $\left(\mathrm{mW} \mathrm{m}^{-2}\right)$ & 1.08 & 1.08 & 1.08 & 1.08 & 1.08 \\
\hline Low value & $0.25 \Omega^{-1}$ & $2.5 \times 10^{5} \mathrm{~m}^{-3}$ & $50 \mathrm{eV}$ & $2.5 \mathrm{~km} / \mathrm{s}$ & $2 R_{\mathrm{Io}}$ \\
\hline Peak parallel potential $(\mathrm{kV})$ & 0.50 & 2.90 & 1.51 & 0.39 & 2.49 \\
\hline Peak energy flux $\left(\mathrm{mW} \mathrm{m}^{-2}\right)$ & 0.30 & 1.53 & 1.13 & 0.21 & 2.60 \\
\hline
\end{tabular}

density in the torus $\left(\rho_{T}\right)$, and the ionospheric conductivity $\left(\Sigma_{P}\right)$.

[39] As in the baseline case (Figure 5c), the upward parallel current (Figure 8c) is restricted by the currentvoltage relation, primarily that of the low-density plasma $\sim 2 R_{J}$ from Jupiter's center. This restricted parallel current leads to a radial current (Figure 8e) that is not similar in shape to the imposed electric field, so the $K_{T} \times B_{T}$ force is not self-similar. As a result, the decay of the velocity is not self-similar. In spite of the non-self-similar decay, the parallel potential and energy flux decay nearly exponentially in time, but at a somewhat slower rate than predicted by a simple model with no parallel electric field. Again, the decay rate is sensitive to $\Sigma_{P}$ and $\rho_{T}$, so the model is in agreement with the observed length of the trail. The modification of $\Sigma_{P}$ by precipitating electrons (Figure $8 \mathrm{f}$ ) has almost no effect.

[40] The solutions displayed in Figure 8 indicate that the finite pressure effects in the magnetosphere are expected to change the detailed shape of the initial velocity profile but have the effect of enhancing the potential and the energy flux by less than a factor of two. Otherwise, the trail aurora and its characteristics are qualitatively similar to those predicted in Figures 3 and 5.

\section{Discussion and Conclusions}

[41] The above model follows previous work of Su et al. [2003] and Delamere et al. [2003], who suggest that the trail or wake aurora is from the subcorotating plasma in Io's wake created by the momentum exchange of Io with the Io torus plasma. Su et al. [2003] examined the flux tube between the Io torus and Jupiter and concluded that the current-voltage relation was dominated by the low-density region $\sim 2 R_{J}$ from Jupiter's center. Using height-integrated conductivity in Jupiter's ionosphere and height-integrated properties of the Io torus, our model is based on a set of six nonlinear equations that couple the Io torus plasma to Jupiter's ionosphere. With a modified current voltage relation, we solve for a quasi-static solutions as a function of radial distance from Io's orbit assuming that variations in time and the $\varphi$ direction are small. The model assumes a dipole magnetic field and ignores variations in $\rho_{T}$ and $B_{T}$ along Io's orbit.

[42] The numerical solutions predict that, under the current-voltage relation derived from Ray et al. [2009], a parallel electric field will develop on the order of $1 \mathrm{kV}$ of potential resulting in an $\sim 1 \mathrm{~mW} \mathrm{~m}$ m $^{-2}$ of electron energy flux. The wake trail is expected to be roughly $1 / 8$ to $1 / 4$ of the circumference of the Io torus. These solutions are, within error, in good agreement with the observed emission signatures lending credence to the basic idea that the trail aurora is primarily from subcorotating plasma in the Io torus.

[43] Of critical importance is the modified current-voltage relation [Ray et al., 2009]. The plasma in Jupiter's ionosphere is gravitationally bound and the plasma in the Io torus, mostly $\mathrm{O}^{+}$and $\mathrm{S}^{++}$, is bound to the Io torus by a centrifugal potential from Jupiter's rapid rotation. The

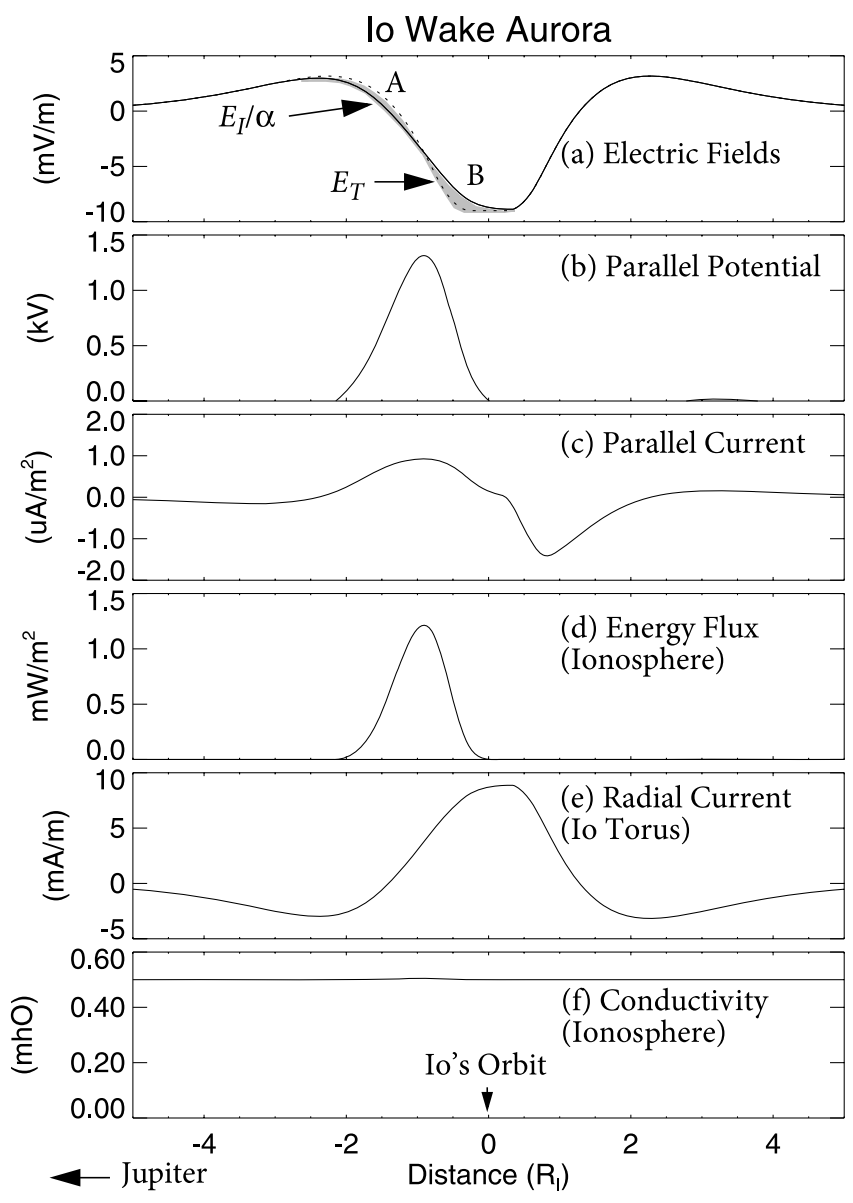

Figure 8. The results of a model with parallel electric fields and a more complex initial state of subcorotation. (a) Ionospheric and magnetospheric electric fields, (b) the parallel potential, (c) field-aligned currents, (d) the electron energy flux into Jupiter's ionosphere, (e) the heightintegrated radial current in the Io torus, and (f) the ionospheric conductivity as a function of radial position from Io's orbit. 
plasma density at high latitudes, roughly $2 R_{J}$ from Jupiter's center, is essentially controlled by the amount of $\mathrm{H}^{+}$in Jupiter's magnetosphere. We call this effect the "highlatitude current choke." The current-voltage relation between the Io torus and Jupiter are thus dominated by the plasma properties at $\sim 2 R_{J}$, which is expected to be the region of auroral acceleration. Without this modified current-voltage relation, no significant parallel electric fields are expected to form.

[44] In the case of the Io wake aurora, the formation of the parallel electric fields modifies the detailed structure of the current system, but does not grossly change the relaxation time or angular momentum change between Jupiter and the Io torus. The relaxation time, $\tau=\rho_{T} /\left(2 \alpha \beta \Sigma_{P} B_{T}^{2}\right)$, is controlled by the height-integrated mass of the Io torus and the ionospheric conductivity. The density and structure of the Io torus is fairly well understood [Bagenal, 1994, 1997], so $\rho_{T}$ can be estimated within a factor of two. $B_{T}$ is well constrained. The ionospheric conductivity is less well established, but is believed to be on the order of $\sim 0.5 \Omega^{-1}$, with a possible factor of 4 range. Our solutions indicated that the best match to the length of Io's trail is $\Sigma_{P} \sim 0.2 \Omega^{-1}$ to $0.5 \Omega^{-1}$, values that are very close to that predicted by atmospheric models.

[45] A scale size of the wake aurora (as measured in the Io torus) was derived using several simplifying assumptions. This scale size appears in the numerical solutions as an exponential relaxation of the potential and electric fields as a function of distance from the region of maximum parallel potential. In the case of the Io wake aurora this scale size roughly $1 R_{I o}$, leads to an arc width on the order of $\sim 100-1000 \mathrm{~km}$ at Jupiter.

[46] These same set of equations, with minor modification, can be applied to analyze the behavior of the main auroral oval. Earlier attempts [e.g., Nichols and Cowley, 2003, 2004, 2005] use a relaxation method which may not fully reveal the effects of the parallel electric field. In fact, the application of Faraday's law predicts that the ionospheric rotation rate must lag the magnetospheric rotation rate in order to develop a quasi-static parallel potential (equation (11)). Such a nonlinear and somewhat counterintuitive effect does not emerge from relaxation techniques [Nichols and Cowley, 2005]. Furthermore, the high-latitude current choke can severely impact the currents at $\sim 20 R_{J}$, so use of the magnetospheric parameters will lead to inaccurate predictions for the main aurora. Use of the fully nonlinear set of equations is needed to investigate more accurately the impact of the parallel electric fields on the main auroral oval.

[47] Acknowledgments. The authors wish to thank Laila Andersson for useful input.

[48] Wolfgang Baumjohann thanks Robert Lysak and another reviewer for their assistance in evaluating this paper

\section{References}

Bagenal, F. (1994), Empirical model of the Io plasma torus: Voyager measurements, J. Geophys. Res., 99, 11,043.

Bagenal, F. (1997), Ionization source near Io from Galileo wake data, Geophys. Res. Lett., 24, 2111.

Boström, R. (2003), Kinetic and space charge control of current flow and voltage drops along magnetic flux tubes: Kinetic effects, J. Geophys. Res., 108(A4), 8004, doi:10.1029/2002JA009295.
Chust, T., A. Roux, W. S. Kurth, D. A. Gurnett, M. G. Kivelson, and K. K. Khurana (2005), Are Io's Alfvén wings filamented? Galileo observations, Planet. Space Sci., 53, 395.

Clarke, J. T., et al. (1996), Far-ultraviolet imaging of Jupiter's aurora and the lo "footprint", Science, 274, 404

Clarke, J. T., L. Ben Jaffel, and J.-C. Gérard (1998), Hubble Space Telescope imaging of Jupiter's UV aurora during the Galileo orbiter mission, J. Geophys. Res., 103, 20,217.

Clarke, J. T., et al. (2002), Ultraviolet emissions from the magnetic footprints of Io, Ganymede and Europa on Jupiter, Nature, 415, 997.

Connerney, J. E. P., R. Baron, T. Satoh, and T. Owen (1993), Images of excited $\mathrm{H}^{+}$at the foot of the Io flux tube in Jupiter's atmosphere, Science, 262,1035

Crary, F. J. (1997), On the generation of an electron beam by Io, J. Geophys. Res., 102, 37.

Delamere, P. A., F. Bagenal, R. E. Ergun, and Y.-J. Su (2003), Momentum transfer between the Io plasma wake and Jupiter's ionosphere, J. Geophys. Res., 108(A6), 1241, doi:10.1029/2002JA009530.

Ergun, R. E., C. W. Carlson, J. P. McFadden, F. S. Mozer, and R. J. Strangeway (2000), Parallel electric fields in discrete arcs, Geophys. Res. Lett., 27, 4053.

Ergun, R. E., Y.-J. Su, L. Andersson, F. Bagenal, P. A. Delamere, R. L. Lysak, and R. J. Strangeway (2006), S-bursts and the Jupiter ionospheric Alfvén resonator, J. Geophys. Res., 111, A06212, doi:10.1029/ 2005JA011253.

Gérard, J.-C., J. Gustin, D. Grodent, P. Delamere, and J. T. Clarke (2002), The excitation of the FUV Io tail on Jupiter: Characterization of the electron precipitation, J. Geophys. Res., 107(A11), 1394, doi:10.1029/ 2002JA009410.

Goertz, C. K. (1980), Io's interaction with the plasma torus, J. Geophys. Res., 85, 2949.

Goldreich, P., and D. Lynden-Bell (1969), Io, a Jovian unipolar inductor, Astrophys. J., 156, 59.

Grodent, D., J. T. Clarke, J. Kim, J. H. Waite Jr., and S. W. H. Cowley (2003), Jupiter's main oval observed with HST-STIS, J. Geophys. Res., 108(A11), 1389, doi:10.1029/2003JA009921.

Hill, T. W. (1979), Inertial limit on corotation, J. Geophys. Res., 84, 6554 Hill, T. W., and V. M. Vasyliunas (2002), Jovian auroral signature of Io's corotational wake, J. Geophys. Res., 107(A12), 1464, doi:10.1029/ 2002JA009514.

Kivelson, M. G., K. K. Khurana, R. J. Walker, J. Warnecke, C. T. Russell, J. A. Linker, D. J. Southwood, and C. Polanskey (1996), Io's interaction with the plasma Torus: Galileo magnetometer report, Science, 274, 396 Knight, S. (1973), Parallel electric fields, Planet Space Sci., 21, 741.

Lyons, L. R. (1980), Generation of large-scale regions of auroral currents, electric potentials, and precipitation by the divergence of the convection electric field, J. Geophys. Res., 85, 17.

Lyons, L. R. (1981), Discrete aurora as the direct result of an inferred highaltitude generating potential distribution, J. Geophys. Res., 86, 1.

Lyons, L. R., D. S. Evans, and R. Lundin (1979), An observed relation between magnetic field-aligned electric fields and downward energy fluxes in the vicinity of auroral forms, J. Geophys. Res., 84, 457.

Millward, G., S. Miller, T. Stallard, A. D. Aylward, and N. Achilleos (2002), On the dynamics of the jovian ionosphere and thermosphere III, The modelling of auroral conductivity, Icarus, 160, 95.

Nichols, J. D., and S. W. H. Cowley (2003), Magnetosphere-ionosphere coupling currents in Jupiter's middle magnetosphere: Dependence on the effective ionospheric Pedersen conductivity and iogenic plasma mass outflow rate, Ann. Geophys., 21, 1419.

Nichols, J. D., and S. W. H. Cowley (2004), Magnetosphere-ionosphere coupling currents in Jupiter's middle magnetosphere: Effect of precipitation-induced enhancement of the ionospheric Pedersen conductivity, Ann. Geophys., 22, 1799.

Nichols, J. D., and S. W. H. Cowley (2005), Magnetosphere-ionosphere coupling currents in Jupiter's middle magnetosphere: Effect of magnetosphere-ionosphere decoupling by field-aligned auroral voltages, Ann. Geophys., 23, 799.

Paschmann, G., S. Haaland, and R. Treumann (2003), Auroral Plasma Physics, 1st ed., Kluwer Acad., Dordrecht, Netherlands.

Pontius, D. H., Jr., and T. W. Hill (1982), Departure from corotation of the Io plasma torus: Local plasma production, Geophys. Res. Lett., 9(12), 1321.

Ray, L. C., Y.-J. Su, R. E. Ergun, P. A. Delamere, and F. Bagenal (2009), Current-voltage relation of a centrifugally confined plasma, J. Geophys. Res., doi:10.1029/2008JA013969, in press.

Saur, J. (2004), A model of Io's local electric field for a combined Alfvénic and unipolar inductor far-field coupling, J. Geophys. Res., 109, A01210, doi:10.1029/2002JA009354. 
Su, Y.-J., R. E. Ergun, F. Bagenal, and P. A. Delamere (2003), Io-related Jovian auroral arcs: Modeling parallel electric fields, J. Geophys. Res., 108(A2), 1094, doi:10.1029/2002JA009247.

Su, Y.-J., S. T. Jones, R. E. Ergun, F. Bagenal, S. E. Parker, P. A. Delamere, and R. L. Lysak (2006), Io-Jupiter interaction: Alfvén wave propagation and ionospheric Alfvén resonator, J. Geophys. Res., 111, A06211, doi:10.1029/2005JA011252.

F. Bagenal, P. A. Delamere, V. Dols, R. E. Ergun, and L. Ray, Laboratory for Atmospheric and Space Physics, University of Colorado, Boulder, CO 80303, USA. (robert.ergun@colorado.edu)

Y.-J. Su, Department of Physics, University of Texas at Arlington, Arlington, TX 76019, USA. 